\title{
Effectiveness of Progressive Muscle Relaxation Technique among the Antenatal Mothers above 32 Weeks of Gestation on the Reactivity and Time Consumption of Non Stress Test: A Randomized Controlled Trial
}

\author{
L. Ngahneilam ${ }^{1}$, Sukhjit Kaur ${ }^{1}$, Karobi Das ${ }^{1}$, Pradip Kumar Saha ${ }^{2}$ \\ ${ }^{1}$ National Institute of Nursing Education, PGIMER, Chandigarh \\ ${ }^{2}$ Additional Professor, Department of Obstetrics and Gynaecology, PGIMER, Chandigarh
}

Corresponding Author: L. Ngahneilam

\begin{abstract}
Background: Non Stress Test is a simple, inexpensive and non-invasive method to assess the wellbeing of the fetus by observing the FHR with its acceleration in response to the movement of the fetus.

Objective: To assess the effectiveness of progressive muscle relaxation technique among the antenatal mothers above 32 weeks of gestation on reactivity and time consumption of Non Stress Test.

Design: Randomized controlled trial

Setting: Obstetrics and Gynaecology OPD, PGIMER, Chandigarh

Participant: 120 Antenatal mothers $\geq 32$ weeks of gestation

Methods: 120 pregnant mothers i.e. sixty each in Experimental and Control group willing to participate and available at the time of data collection were enrolled through a random sampling technique. Progressive muscle relaxation technique was demonstrated and was performed simultaneously by the antenatal mothers 15 to 20 minutes prior to NST who were enrolled under the Experimental group. Routine care was given to Control group. Data were collected by using an interview schedule in the month of October to December 2020. Non stress test was done as per schedule of antenatal visit and interpretation of NST graph in relation to the reactivity, time consumption and baseline fetal heart rate were compared in both the group.
\end{abstract}

Results: It revealed that all the antenatal mothers in the Exp. group and 90\% of mothers in the Control group showed reactivity of Non stress test. In relation to time consumption of Non stress test, all mothers in the Experimental group took normal time i.e. 20 minutes. Out of the $90 \%$ of antenatal mothers who were reactive in the Control group, $5 \%$ took more than 20 minutes. A statistically significant difference was found in relation to reactivity by applying Chi Square $(\mathrm{p}<0.05)$. The finding also showed a significant difference in between the Experimental and Control group in relation to the reactivity of Non stress test as shown by Mann Whitney U test, baseline fetal heart rate during NST, all the antenatal mothers had normal BHR between 110 to $160 \mathrm{bpm}$ in both groups.

Conclusion: It can be concluded that Progressive muscle relaxation technique performed prior to Non stress test can be used for improving the Non stress test results, timesaving, evokes positive feeling and satisfaction among the antenatal mothers.

Key Words: Non Stress Test, Progressive Muscle Relaxation Technique, Reactivity

\section{INTRODUCTION}

Pregnancy is a natural process and not an illness but there are progressive anatomical, physiological and psychological changes that occurs during the course of pregnancy. The length of human pregnancy 
L. Ngahneilam et.al. Effectiveness of progressive muscle relaxation technique among the antenatal mothers above 32 weeks of gestation on the reactivity and time consumption of non stress test: a randomized controlled trial.

is usually 40 weeks. First trimesters are up to 12 weeks, second from 13 to 28 weeks and third from $29^{\text {th }}$ to 40 weeks ${ }^{1}$

The physiological changes that take place during pregnancy are adaptations in which the pregnant woman's body undergoes for accommodating the growing embryo or fetus. These changes are cardiovascular, metabolic and systemic changes i.e. respiratory, urinary, alimentary, nervous system. During the course of pregnancy, the cardiovascular system goes through important structural and hemodynamic changes. These adaptations enable fetal growth and development, and maladaptation during this period has been associated with fetal morbidity. By the $16^{\text {th }}$ week of gestation, the first movement or momentous kicks which is called quickening may be felt by the mother. By the $20^{\text {th }}$ weeks, fetal movement is mostly felt by the mothers. The fetal kicks will become stronger and regular at the $28^{\text {th }}$ weeks. As the fetus grows, the movement will become obvious, it will be gradually felt more regular. The fetal movement's frequency increases to until $32^{\text {nd }}$ weeks from the $24^{\text {th }}$ weeks period of gestation. The frequency of the movement of fetus inclines to plateau until the onset of labour from $32^{\text {nd }}$ weeks ${ }^{2}$

Antenatal care is one of the significant components for ensuring a normal pregnancy to deliver a healthy baby from a healthy mother. Prenatal care does not guarantee a normal baby, but it can identify the problems early so that they can be minimized or eliminated. ${ }^{3}$ Throughout pregnancy fetal well-being monitoring during the first trimester is done by transvaginal sonography, nuchal translucency, crown to rump length and maternal serum markers like the maternal serum alpha fetoprotein (MSAFP), triple test, human chorionic gonadotrophins, unconjugated oestriol, acetylcholinesterase, Inhibin A. During second-trimester maternal serum markers, ultrasonography, chorionic villi sampling, amniocentesis. Biochemical, physiological and physical surveillance techniques can be used to monitor fetal well being especially in the third trimester. During the antenatal visit, antenatal examination includes auscultation of fetal heart rate. ${ }^{2}$

The process of FHR monitoring is known as NST. NST is a relatively inexpensive procedure with no contraindication or side effect. The appropriate time to perform NST is after 32 weeks of gestation. During NST the mother is given a comfortable position preferably left lateral. Women are asked to empty the bladder and should not be on empty stomach before the test. The ultrasound transducer is placed over the abdomen after auscultation of fetal heart rate. Non stress test is usually performed for 20 minutes but if expected results are not seen it may be extended up to 40 minutes. Based on the test, it is classified as reactive and nonreactive. ${ }^{4}$ NST parameter includes BHR, variability, acceleration, decelerations. BHR refers to the average rate between peaks and depressions over a period of time that does not include acceleration or deceleration of the heart rate, the normal basal heart rate 110- 160 beats per minute for 10 minutes segment for a duration of more than two minutes. Baseline variability denotes to the variation of FHR from one beat to the next. The normal variability is between 5 to 25 bpm, minimal variability $<5 \mathrm{bpm}$, moderate variability 5 to $25 \mathrm{bpm}$, maximum variability $>25 \mathrm{bpm}$. An abrupt increase in baseline heart rate is the acceleration i.e. 15 bpm above baseline for $>15$ seconds. There should be at least two accelerations every 20 minutes $^{5}$. Decelerations are a gradual or abrupt decrease in baseline heart rate. Deceleration occurs to a lack of oxygenation in the fetus. Early decelerations are gradual fall in the fetal heart rate which start when uterine contractions begin and recover when uterine contractions stop. Variable decelerations are usually caused by umbilical cord compression. Late decelerations start at the height of a uterine contraction and recuperate after the compression ends. A deceleration that lasts between two to three minutes is classified as 
L. Ngahneilam et.al. Effectiveness of progressive muscle relaxation technique among the antenatal mothers above 32 weeks of gestation on the reactivity and time consumption of non stress test: a randomized controlled trial.

non reassuring and if it takes lengthier than 3 minutes it is instantaneously classified as abnormal. Action must be taken quickly e.g. emergency caesarean section. Non reactive traces are associated with neonatal deaths, IUGR, intrauterine hypoxia. ${ }^{6,7}$

If Non stress test shows non reactive results in 20 minute, the test is extended up to 40 minutes and again if the results are the same, the antenatal mother is sent for a further biophysical profile. So, a Non stress test does not imply a problem, it could be due to some factor, so further evaluation is needed. Certain factors that influence the reactivity and time in achieving the reactivity during NST i.e. position of the antenatal mother, type of diet, exercise, manual and vibroacoustic stimulation, baby's sleep cycle, sedative drugs, fear, stress, anxiety ${ }^{8}$. Studies have also shown the effects of music, acupressure, maternal position, gestational age on the result of NST. Non-pharmacologic interventions such as hypnosis, visual imagery, yoga, and relaxation, can reduce the anxiety to both the mother and fetus. Muscle relaxation technique reduces the sympathetic anxiety symptoms and one can feel comfortable. ${ }^{9}$

However, the present study concentrates on the effectiveness of progressive muscle relaxation technique on the reactivity and time consumption of NST.

\section{MATERIAL AND METHODS}

A RCT designed was adopted to assess the effectiveness of progressive muscle relaxation technique among the antenatal mothers on reactivity and time consumption of NST. Antenatal mothers were grouped into the experimental and control group in random manner. Antenatal mothers in the control group received the routine care and antenatal mothers in the experimental received the Progressive muscle relaxation technique 10 to 15 minutes prior to NST. The outcome was assessed on the NST result for both the group

\begin{tabular}{|l|l|l|}
\hline Control group (C) & & $\mathrm{O}_{\mathrm{C}}$ \\
\hline Experimental group ( E) & $\mathrm{X}$ & $\mathrm{O}_{\mathrm{E}}$ \\
\hline
\end{tabular}

$\mathrm{C}=$ Control group

$\mathrm{E}=$ Experimental group

$\mathrm{X}=$ Implementation of Progressive muscle relaxation technique

$\mathrm{O}_{\mathrm{c}}=$ Assessment of the Non Stress test result after routine care in the Control group

$\mathrm{O}_{\mathrm{E}}=$ Assessment of Non stress test results in the experimental group after Progressive muscle relaxation technique.

Research setting: The study was conducted in Obs \& Gynae, OPD at PGIMER, Chandigarh, situated at $2^{\text {nd }}$ floor C Block, new OPD building. PGIMER which is one of the premier medical Institutes in India.

\section{Sampling technique:}

- Randomization by using computergenerated random

- Total enumeration sampling was used to enrolled the sample for the study

\section{Randomization:}

- Randomization was prepared by using computer-generated random numbers from Research randomizer i.e. https://www.randomizer.org with an allocation concealment

\section{Inclusion criteria:}

- Antenatal mothers, singleton pregnancy, without any psychological disorder

- No history of smoking, drug abuse

\section{Intervention:}

\section{Control group:}

- Antenatal mothers were explained about the Non stress test procedure to reduce fear, anxiety and enhance cooperation

- The antenatal mothers were asked to empty the bladder prior to the procedure to avoid discomfort during abdominal palpation

- The mother is asked not to be on empty stomach.

- Privacy is provided and made her to lie in a left lateral position, Leopold manoeuvres is performed to determine the fetal back 
L. Ngahneilam et.al. Effectiveness of progressive muscle relaxation technique among the antenatal mothers above 32 weeks of gestation on the reactivity and time consumption of non stress test: a randomized controlled trial.

- Non stress test was performed, monitored for 20 minutes. To those antenatal mothers who were non reactive, it was extended up to 40 minutes

\section{Experimental group:}

- Antenatal mother was made to sit in a comfortable chair in a calm and a conducive room

- Progressive muscle relaxation technique was demonstrated to the antenatal mothers (deep muscle relaxation technique, which involves the tensing and relaxation of all the major muscles in an orderly manner, from the head to foot).

- Simultaneously the antenatal mothers performed the progressive muscle relaxation technique (10 to 15 minutes).

- Non stress test was performed (following the same steps of procedure as performed for the control group).

\section{Outcomes and measurements:}

The antenatal mothers' sociodemographic data and obstetrical data were taken after receiving consent. Outcome measured included reactivity and time consumption of the NST.

The material used for measuring the outcome was:

- Non stress test graphical paper

The following parameters were assessed. The description of each parameter is as follows:

- Baseline heart rate: The average rate between peaks and depressions for a duration of more than 2 minutes in a 10minute segment, that does not include accelerations or decelerations. The normal BHR is 110 to $160 \mathrm{bpm}$.

- Variability: Normal variability is between 5 to 25 beats per minute.

- Acceleration: It refers to a visually apparent abrupt increase in fetal heart rate and peak which must be $\geq 15 \mathrm{bpm}$ for 15 seconds of an antenatal mother above 32 weeks of gestation.

- Time consumption: Total time taken to achieve two accelerations during Non stress test $^{3}$

\section{Data collection method:}

1) Interview schedule

2) Observation - Baseline FHR, fetal heart rate acceleration on graph paper, variability, time taken for 2 acceleration, the reactivity of NST result,

\section{Procedure for data collection}

- Data was collected from Obstetrics and Gynaecology OPD, PGIMER, Chandigarh.

- Antenatal mothers were randomly allocated into the experimental and control group using computer-generated random numbers with allocation concealment.

- Antenatal mothers were interviewed and proforma containing a sociodemographic profile, clinical profile and Non stress test record.

- Progressive muscle relaxation technique was demonstrated and simultaneously performed by the experimental group for 10 to 15 minutes. The control group received routine care.

- Non stress test was done on both the group and NST graph was observed for reactivity, time consumptions and baseline heart rate and variability.

\section{Ethical considerations}

- Written permission was obtained from the concerned authority - the Head of Department of Obstetrics and Gynaecology PGIMER, Chandigarh.

- Ethical approval was taken from the ethical committee of the institution vide no. NK/6008/MSc/301

- The doctors and the nursing officers of the Obstetrics and Gynaecological OPD were informed about the study.

- Written informed consent was taken from the antenatal mothers 
L. Ngahneilam et.al. Effectiveness of progressive muscle relaxation technique among the antenatal mothers above 32 weeks of gestation on the reactivity and time consumption of non stress test: a randomized controlled trial.

- Confidentiality was maintained throughout the study

- Women were assured that the information given by them will be kept confidential and used only for research purpose.

- During data collection, there was no interruption in the routine care and activities of the antenatal mother

- This study was beneficial for the antenatal mothers in achieving a better result of NST

\section{Permission for study:}

Written permission for the study was obtained from the Head of Obstetrics and Gynaecology OPD, PGIMER, Chandigarh

A pilot study was conducted before the actual data collection period in Obstetrics and Gynaecology OPD, PGIMER, Chandigarh July 2020. It was conducted to assess the feasibility of this study with the objectives of:

- To assess the availability of subjects.

- To assess the validity, reliability and practicability of research tools.

- To assess the procedural deficiencies in methodology.

- To estimate the time required for each study subject.

The changes that were needed to be made were incorporated after the pilot study

\section{Data analysis and interpretation:}

All analysis were performed using SPSS version 20. Descriptive statistics such as frequency distribution table, percentages, mean (SD) and comparison between the group were used. In inferential statistics, Chi-square test, fisher's exact test and Mann Whitney U test were used as the data did not follow a normal distribution. Findings of the study was presented with the help of tables and figures.

Effectiveness of progressive muscle relaxation technique among the Antenatal mothers above 32 weeks of gestation on reactivity and time consumption of Non stress test was assessed by doing statistical analysis of data by SPSS version 20 and Microsoft excel. Analysed data was presented in the form of tables, graphs and figures.

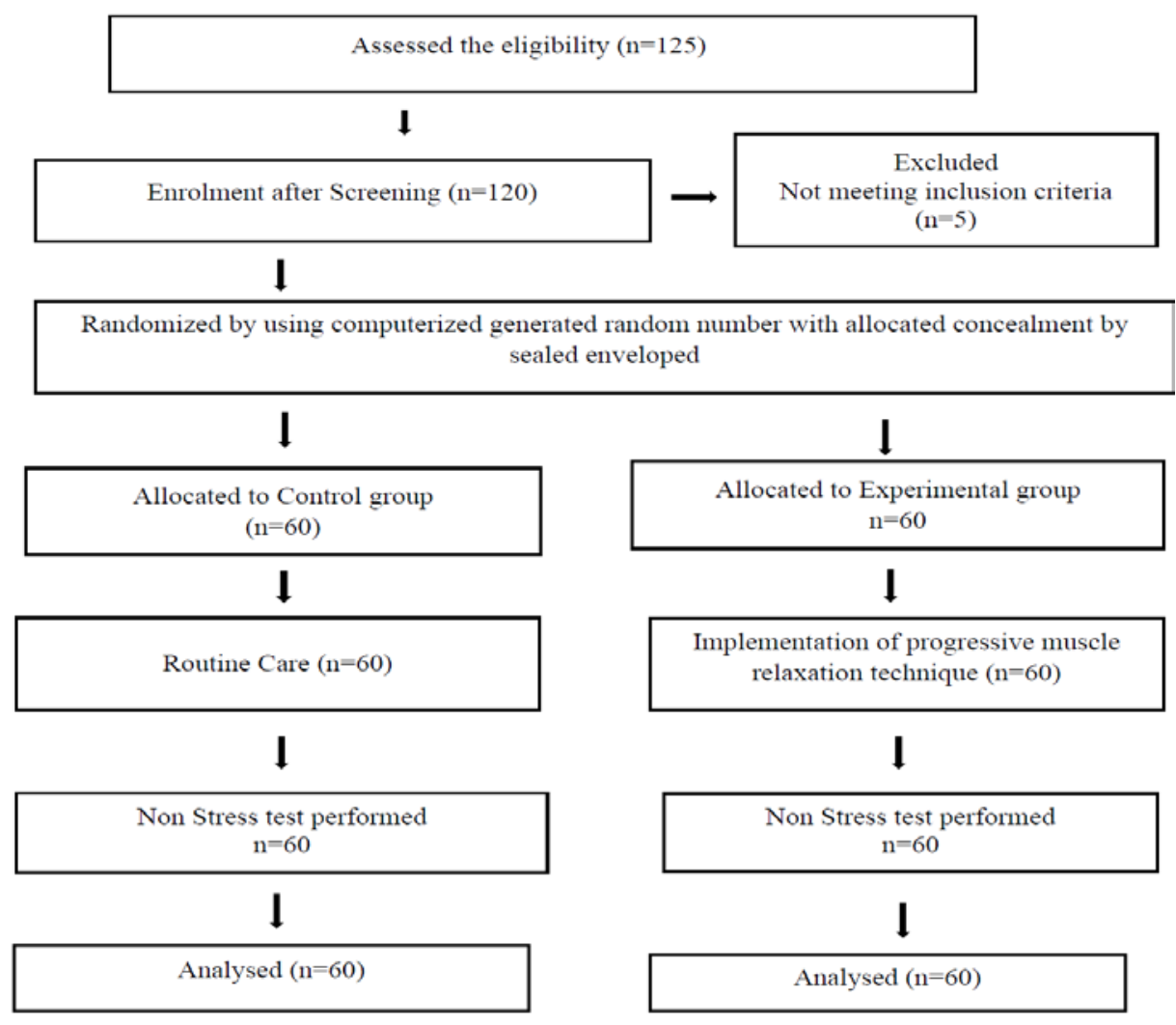

Fig. 1: Consort Diagram 
L. Ngahneilam et.al. Effectiveness of progressive muscle relaxation technique among the antenatal mothers above 32 weeks of gestation on the reactivity and time consumption of non stress test: a randomized controlled trial.

\section{RESULT}

The socio-demographic variables of the Antenatal mothers in the Experimental and Control group are depicted in table 1. The age group of 26-30 years had the maximum no. of Antenatal mothers in both the groups, $50 \%$ were in the Control group and $46.7 \%$ in the Experimental group, Mean $\pm \mathrm{SD}=28.94 \pm .1$ (years), Range $=20-40$ years. Majority of Antenatal mothers in both the groups were graduates, homemakers from Hindu religion and joint families with $48.3 \%$ belonging to the upper class in the Experimental group and $56.7 \%$ of the Control group in the upper-middle-class.

Table 1: Socio-demographic profile of Antenatal mothers, $N=120$

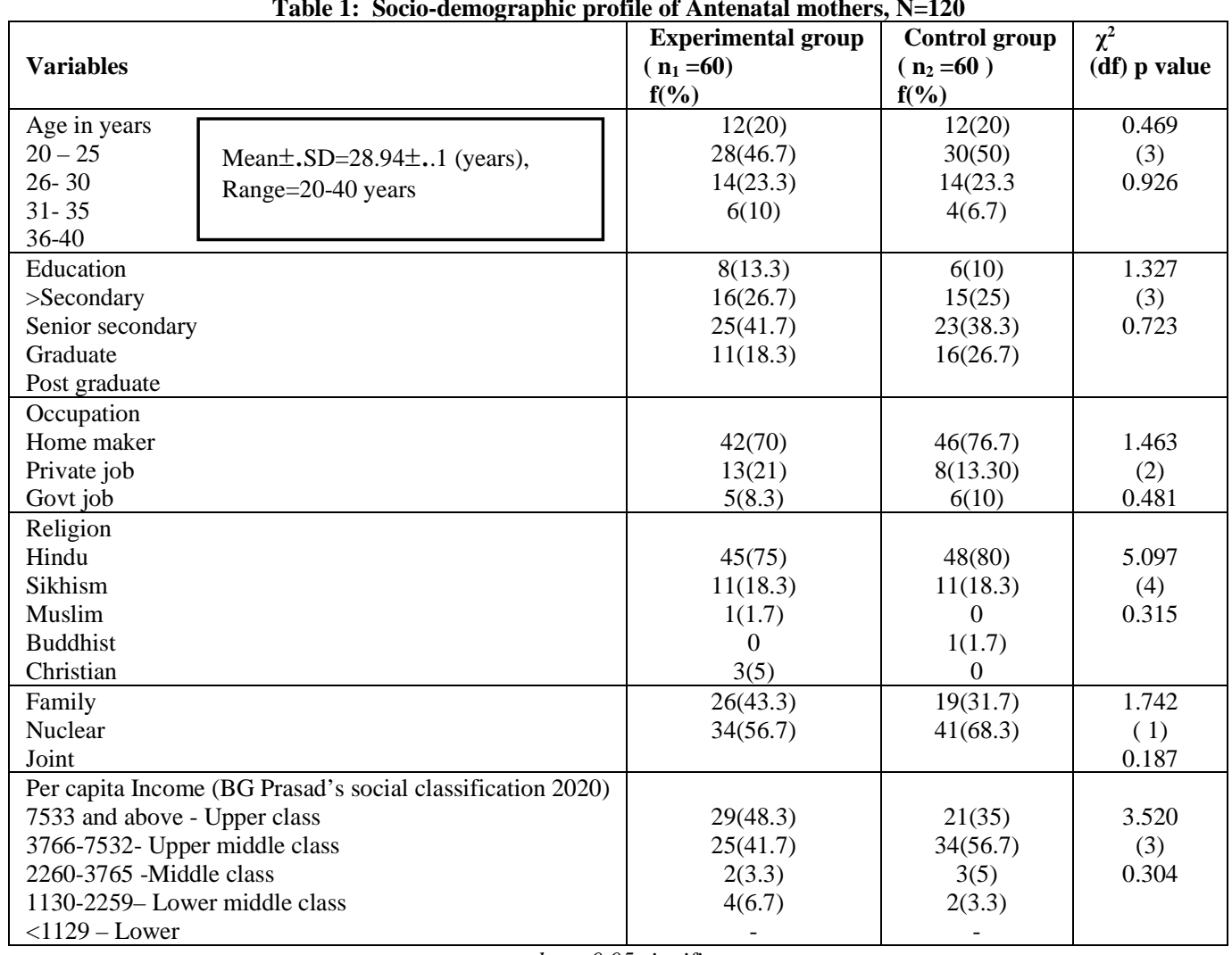

p-value $<0.05$ significant

Obstetrics finding of antenatal mothers are depicted in table 2. More than 93.3\% of the presentation were cephalic and longitudinal in both the groups, less than $6.7 \%$ of the antenatal mother had malpresentation and transverse lie in both the groups. More than $55 \%$ of the antenatal mother's fetal head were not engaged in both the groups. All antenatal mothers felt fetal movement except one antenatal mother in the Control group.

Table 2: Obstetric examination findings of Antenatal mothers, $N=120$

\begin{tabular}{|c|c|c|c|}
\hline Variables & Experimental group $\left(n_{1}=60\right) f(\%)$ & Control group $\left(n_{2}=60\right)$ f (\%) & $\chi^{2}$ (df) $p$ value \\
\hline \begin{tabular}{ll}
\multicolumn{2}{l}{ Presentation } \\
- & Cephalic \\
- & Malpresentation \\
\end{tabular} & $\begin{array}{c}56(93.3) \\
4(6.7)\end{array}$ & $\begin{array}{c}57(95) \\
3(5)\end{array}$ & $\begin{array}{c}0.152 \\
(1) \\
1.000 \\
\end{array}$ \\
\hline $\begin{array}{ll}\text { Lie } & \\
\text { - } & \text { Transverse } \\
\text { - } & \text { Longitudinal } \\
\end{array}$ & $\begin{array}{c}3(3.3) \\
57(95.0)\end{array}$ & $\begin{array}{c}2(3.3) \\
58(96.7)\end{array}$ & $\begin{array}{c}0.209 \\
(1) \\
1.000 \\
\end{array}$ \\
\hline \begin{tabular}{ll}
\multicolumn{2}{l}{ Engagement of fetal head } \\
- & No \\
- & Yes \\
\end{tabular} & $\begin{array}{l}33(55) \\
27(45) \\
\end{array}$ & $\begin{array}{l}38(63.3) \\
22(36.7)\end{array}$ & $\begin{array}{c}0.862 \\
(1) \\
0.352 \\
\end{array}$ \\
\hline $\begin{array}{l}\text { Fetal movement } \\
\text { - } \quad \text { Present } \\
\text { - } \quad \text { Absent }\end{array}$ & $\begin{array}{c}60(100) \\
0\end{array}$ & $\begin{array}{c}59(98.4) \\
1(1.7)\end{array}$ & $\begin{array}{l}1.008 \\
(1) \\
1.00\end{array}$ \\
\hline
\end{tabular}

p-value $<0.05$ significant 
L. Ngahneilam et.al. Effectiveness of progressive muscle relaxation technique among the antenatal mothers above 32 weeks of gestation on the reactivity and time consumption of non stress test: a randomized controlled trial.

Table 3 Illustrates all the antenatal mothers who were reactive within 20 minutes in the Experimental group, out of $90 \%$, who were reactive in the control group, $85 \%$ were reactive within 20 minutes. All the antenatal mothers had a normal BHR

Table 3: Comparison of Non stress test results, $\mathrm{N}=120$

\begin{tabular}{|l|l|l|}
\hline Variables & $\begin{array}{l}\text { Experimental } \\
\text { group } \\
\mathbf{n}_{\mathbf{1}}=\mathbf{6 0} \mathbf{f ( \% )}\end{array}$ & $\begin{array}{l}\text { Control group } \\
\mathbf{n}_{\mathbf{2}}=\mathbf{6 0} \mathbf{f}(\%)\end{array}$ \\
\hline Reactive Non Stress test & $60(100)$ & $54(90)$ \\
\hline $\begin{array}{l}\text { Time Consumption for } \\
\text { reactivity within 20 minute }\end{array}$ & $60(100)$ & $51(85)$ \\
\hline $\begin{array}{l}\text { Normal Baseline fetal heart } \\
\text { rate }\end{array}$ & $60(100)$ & $60(100)$ \\
\hline
\end{tabular}

The comparison of Non stress test reactivity in both the group is shown in table 4. Chi-square was applied to see the statistical significant difference between both the groups, which shows the significant difference as the p-value $<0.05$ hence it proves that Progressive muscle relaxation technique has an impact on the reactivity of Non stress test

Table 4: Effectiveness of Progressive muscle relaxation technique on the Reactivity of Non stress test in both the groups, $\mathrm{N}=120$

\begin{tabular}{|c|c|c|c|}
\hline Variables & $\begin{array}{l}\text { Experimental } \\
\text { group } \\
\left(n_{1}=60\right) f(\%)\end{array}$ & $\begin{array}{l}\quad \text { Control } \\
\text { group } \\
\left(n_{2}=60\right) f(\%)\end{array}$ & $\begin{array}{l}\chi^{2} \\
\text { (df) p } \\
\text { value }\end{array}$ \\
\hline $\begin{array}{l}\text { Reactivity } \\
\text { Reactive } \\
\text { Non reactive }\end{array}$ & $\begin{array}{c}60(100) \\
0\end{array}$ & $\begin{array}{c}54(90) \\
6(10)\end{array}$ & $\begin{array}{c}6.316^{\mathrm{a}} \\
(1) \\
0.027^{\mathrm{b}}\end{array}$ \\
\hline
\end{tabular}

Median (IQR) values are presented in table 5 in relation to time consumption, baseline fetal heart rate, baseline variability and frequency of acceleration. All of these parameter were statistically significant by applying Mann Whitney $U$ test with p value $<0.05$.

Table 5: Effectiveness of Progressive muscle relaxation technique on time consumption for reactivity, Baseline fetal heart rate, Baseline variability, frequency of acceleration of Non stress test result in both the groups, $N=120$

\begin{tabular}{|c|c|c|c|}
\hline Variable & $\begin{array}{l}\text { Experimental group } \\
\left(\mathrm{n}_{1}=60\right) \\
\text { Median (IQR) }\end{array}$ & $\begin{array}{l}\text { Control group } \\
\left(\mathbf{n}_{2}=60\right) \\
\text { Median(IQR) } \\
\end{array}$ & $\begin{array}{l}\text { Mann Whitney } \\
\text { U value } \\
\text { (z value) p value } \\
\end{array}$ \\
\hline Time consumption $\quad$ (Minute) & $6(6-13)$ & $12(8-15)$ & $\begin{array}{c}959 \\
(-3.919) \\
0.001 \\
\end{array}$ \\
\hline Baseline fetal heart rate & $132(130-136)$ & $140(132-142)$ & $\begin{array}{c}1052 \\
(-3.944) \\
0.001 \\
\end{array}$ \\
\hline Baseline variability (bpm) & $20(15-23.5)$ & $15(11.25-23)$ & $\begin{array}{c}1409 \\
(-2.060) \\
0.039 \\
\end{array}$ \\
\hline $\begin{array}{l}\text { Frequency of acceleration } \\
\text { ( in } 20 \text { minutes) }\end{array}$ & $3(3-4)$ & $3(2-3)$ & $\begin{array}{c}1125.50 \\
(-3.107) \\
0.002 \\
\end{array}$ \\
\hline
\end{tabular}

\section{DISCUSSION}

Antenatal care is one of the significant aspects for ensuring normal pregnancy to deliver a healthy baby. It benefits both the mother and baby. ${ }^{1}$ Non stress test is the most common method of antepartum fetal surveillance. It is a simple, inexpensive and non-invasive method by observing the FHR and fetal heart acceleration in corresponding to the fetal movement ${ }^{2}$ in the antenatal clinic, NST is performed as part of the antenatal care for pregnant mothers above 32 weeks of gestation.

The present study was conducted with an objective to implement and assess the effectiveness of PMRT among antenatal mothers attending Obstetrics and gynaecology OPD, PGIMER.

The present study reveals that Progressive muscle relaxation technique has an impact on Non stress test results, as there is statistically significant difference found between the groups in relation to reactivity, time consumption and baseline fetal heart rate. These findings are in agreement with Akbarzade et al (2014) on the Effect of Maternal Relaxation training on Non stress test, who concluded that relaxation can improve the NST results, reduce the FHR, and increase the number of FHR acceleration, so, relaxation is recommended 
L. Ngahneilam et.al. Effectiveness of progressive muscle relaxation technique among the antenatal mothers above 32 weeks of gestation on the reactivity and time consumption of non stress test: a randomized controlled trial.

during the Non stress test ${ }^{9}$ Dolker et al (2019) studied on the effect of music on the NST and maternal anxiety, supports that music increases the NST reactivity and increased the number of acceleration of the antenatal women, as the mean number of acceleration in the control group was lower than that of the experimental group and 98\% of the experimental group were reactive as compared to the control group (82.0\%), He recommended the use of music during Non stress test $^{10}$ Similar study also done by Kucukkelepce et al (2018) concluded that music played during the Non stress test increased the number of accelerations and improved the reactivity of NST. He has also recommended the use of music during NST for a better reactivity ${ }^{11}$ A related study reported by Khodakarami et al (2020) on the effect of Music on the movement of the fetus during NST has found that there is a significant decrease in the basal FHR in the intervention group as compared with the control group, there was an increase in the number of reactive NST in the intervention group $^{12 .}$ In another study by Kafali et al (2010) found a statistically significant difference between the music and no music groups $^{13}$.

A study conducted by Varsha et al (2015) on a comparative study to the reactivity and time consumption of NST among antenatals on sitting and left lateral position, but there was no statistically significant difference on the reactivity of NST. ${ }^{14}$ Alus et al (2007) compared the effects of 4 different positions on NST and concluded that there is statistically significant difference between 4 groups in terms of reactivity, the least reactivity was shown in the supine position. ${ }^{15}$ Another study was done by Essa et al (2018) on the effect of different maternal position concerning comfort and Cardiotocographic patterns of a fetus during NST. They have concluded that the left lateral position showed more comfort and reactive NST than the other positions. They recommended that a simple protocol for NST application need to be developed which should be followed while performing NST $^{16}$ A study conducted by Rachel et al (2015) on Maternal foetal physiological parameters during NST in 2 different position i.e sitting and left lateral to monitor pregnancy: a cross over study found that there was a significant difference in the baseline FHR. ${ }^{17}$ A study conducted by Cito et al. (2005) in determining the maternal positions during (NST) in different weeks of pregnancy influences FHR patterns. NST for a duration of 30 min were performed in mothers with low-risk pregnancies. They concluded that differences were found in heart rate related to age in gestation and maternal position. The minimum length required to record three acceleration were also found to be significant in terms of age in gestation and maternal position. As the pregnancy progresses the time required to record three large acceleration and defined as reactive decreased. ${ }^{18}$

Finding of the present study correspond with the findings of previous research-based evidence. So, we can draw the inferences that relaxation is an important factor to achieve a better result of Non stress test and it should be an essential part of practice guidelines. So that, effective time evaluation of Antenatal mothers could be done. Results of the present study suggest that Progressive muscle relaxation technique is important before undergoing Non stress test to achieve a reactive NST within time.

\section{Limitation of the study}

- There could be other factors that might have affected the reactivity, baseline fetal heart rate, time consumption like fetal sleep cycle and mother's fed state.

\section{Implication for clinical practice}

- The present study could generate the standard protocol of Progressive muscle relaxation technique prior to performing Non stress test

- It enables the nurse in providing a better reactivity of the Non stress test result.

- The findings of the study will provide baseline data for further studies. 
L. Ngahneilam et.al. Effectiveness of progressive muscle relaxation technique among the antenatal mothers above 32 weeks of gestation on the reactivity and time consumption of non stress test: a randomized controlled trial.

- In-service education can be conducted periodically by the nursing educators to prepare skilled nurses who can provide psychological support to the antenatal mothers.

\section{Recommendation}

- Progressive muscle relaxation technique should be taught to all and should be performed routinely prior to performing Non stress test for relaxing the mother for better NST result and saving the time on NST.

- A similar study can be conducted on labouring mother, postnatal mother and other invasive tests during pregnancy.

- A comparative study can be conducted on other complementary technique with Progressive muscle relaxation technique.

\section{CONCLUSION}

NST is one of the most extensively used technique for antepartum evaluation of the fetus. NST result is classified as reactive or non reactive. The result of the present study revealed that Progressive muscle relaxation technique was effective in relation to reactivity ( $\mathrm{p}$-value $<0.05$ ), time consumption ( $p$ value $<0.05$ ) and basal fetal heart rate ( $\mathrm{p}$ value $<0.05$ ).

Hence, the null hypothesis was rejected and the alternate hypothesis was accepted at a 0.05 level of significance. The study finding concluded that, Progressive muscle relaxation technique helps the antenatal mothers to relax before Non stress test, to achieve better results within time.

Funding Statement: National Institute of Nursing Education, PGIMER, Chandigarh

\section{Declaration of conflict of interest: None declared.}

Acknowledgement: None

Ethical Approval: Approved

\section{REFERENCES}

1. Cunningham FG, Lenovo KJ,Bloom S, Hauth JC, Rouse DJ and Spong C .William Ostetrics.23rd edition. Newyork;MC Graw hill;2010

2. Dutta DC. In: Konar H. Textbook of Obstetrics. 7th edition.Kolkata; New Central Agency (P); 2017.

3. Gottfredsdottir H, Steingrímsdóttir $\quad \mathrm{D}$, Björnsdóttir A, Guðmundsdóttir EÝ, Kristjánsdóttir H. Content of antenatal care: does it prepare women for birth?. Midwifery. 2016 Aug 1;39:71-7.

4. Reeder,Martin,,Koniak-Griffin. In : Raman AV. Maternity Nursing family,Newborn, and women's healthcare. 19th edition. New Delhi: Wolters Kluwer (India) Pvt. Ltd; 2018. p.182

5. Bhaskar N. Midwifery and Obstetrical Nursing.3rd Edition.Bangalore: EMMESS Medical Publishers;2019.

6. Bahado Singh R. Mid trimester detection, American journal Obstetrics and Gynaecology.2002.[cited on Oct 2014]; 13(2):86-89 available from URL:http://www.ncbi.nlm.gov/pmc/articles/ PMC2255728.

7. Electronic fetal heart rate monitoring:research guidelines for interpretation. National Institute of Chold Health and Human development Research Planning Workshop. American journal Obstetricss and Gynaecology Dec 1997 [cited on 2014 nov 2] 177(6):1385-90 available from URL:http://www.ncbi.nih.gov/pubmed/2515 29087

8. Umana OD, Siccardi MA. Prenatal Nonstress Test. StatPearls [Internet]. 2020 Feb 13.

9. Akbarzade M, Rafiee B, Asadi N, Zare N. The effect of maternal relaxation training on reactivity of non-stress test, Basal fetal heart rate, and number of fetal heart accelerations: a randomized controlled trial. Int $\mathrm{J}$ Community Based Nurs Midwifery. 2015; 3(1):51-59.

10. Dolker HE, Basar F. The effect of music on the non-stress test and maternal anxiety. Complementary therapies in clinical practice. 2019 May 1;35:259-64.

11. Küçükkelepçe DŞ, Taşhan ST. The effect of music on the results of a non-stress test: A non-randomized controlled clinical trial. 
L. Ngahneilam et.al. Effectiveness of progressive muscle relaxation technique among the antenatal mothers above 32 weeks of gestation on the reactivity and time consumption of non stress test: a randomized controlled trial.

European Journal of Integrative Medicine. 2018 Feb 1;18:8-12.

12. Khodakarami B, Janesari Ladani M, Kazemi F, Aghababaei S. The Effect of Music on Fetus Movement During Non-Stress Test. Avicenna Journal of Nursing and Midwifery Care. 2020 Oct 10;28(4):1-8.

13. Kafalİ $H$, Derbent $A$, Keskİn E, Sİmavli $S$, Gözdemİr E. Effect of maternal anxiety and music on fetal movements and fetal heart rate patterns. The Journal of MaternalFetal \& Neonatal Medicine. 2011 Mar 1;24(3): 461-4.

14. Varsha S, Pardip K. A comparative study to assess the effect of different Maternal position on reactivity and time consumption for non- stress test. Nursing and Midwifery Research Journal October 2015; 144-65

15. Aluş M, Okumuş $H$, Mete $S$, Güçlü $S$. The effects of different maternal positions on non-stress test: an experimental study. Journal of clinical nursing. 2007 Mar; 16(3):562-8

16. Essa RM, Hafaz SK. Effect of Different Positions of Pregnant Women on their Comfort and Fetal Cardiotocographic Patterns during Non Stress Test.
International Journal For Research In Health Sciences And Nursing. 2018 Feb 28;4(2): 01-24.

17. Samuel R, Karkada S, Fernandes S, Bhat P. Materno foetal physiological parameters in sitting and left lateral position during nonstress test (NST) monitoring in pregnancy: a cross over study. Manipal Journal of Nursing and Health Sciences (MJNHS). 2015 Jul 1;1(2):83-6.

18. Cito G, Luisi S, Mezzesimi A, Cavicchioli C, Calonaci G, Petraglia F. Maternal position during non-stress test and fetal heart rate patterns. Acta Obstet Gynecol Scand. 2005;84(4):335-8.

How to cite this article: L. Ngahneilam, Kaur S, Das K et.al. Effectiveness of progressive muscle relaxation technique among the antenatal mothers above 32 weeks of gestation on the reactivity and time consumption of non stress test: a randomized controlled trial. International Journal of Science \& Healthcare Research. 2021; 6(4): 242-251. DOI: https:// doi.org/10.52403/ijshr.20211035 